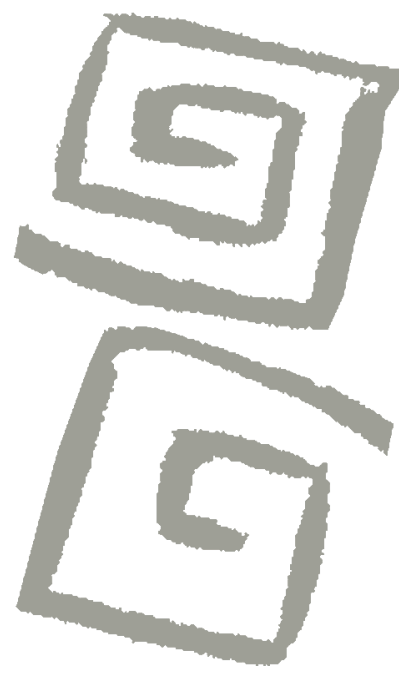

\title{
Aproximación crítica a las políticas públicas en salud indígena, medicina tradicional e interculturalidad en México (1990-2016)
}

\author{
A critical examination of public policies related \\ to indigenous health, traditional medicine, and \\ interculturality in Mexico (1990-2016)
}

Roberto Campos Navarro', Edith Yesenia Peña Sánchez ${ }^{2}$, Alfredo Paulo Maya ${ }^{3}$

'Doctor en Antropología. Profesor titulary coordinador, Posgrado de Antropología en Salud, Universidad Nacional Autónoma de México, México. $\bowtie$ iD

${ }^{2}$ Doctora en Antropología. Investigadora, Instituto Nacional de Antropología e Historia; docente, Posgrado de Antropología en Salud, Universidad Nacional Autónoma de México, México. $₫$ (iD)

${ }^{3}$ Doctor en Antropología. Profesor tiempo completo, Facultad de Medicina, Universidad Nacional Autónoma de México, México. $\square$ (iD)
RESUMEN En los últimos 26 años, el gobierno mexicano desarrolló múltiples discursos y actividades sobre la denominada "salud intercultural" dirigidos, en especial, a los pueblos originarios de México (alrededor de 62, de acuerdo al criterio lingüístico), y construyó establecimientos de salud (puestos de salud, clínicas y hospitales) en los que propuso la aplicación de indicadores de pertinencia cultural en algunos estados como Puebla, Nayarit, Oaxaca, Chiapas, Querétaro y Jalisco (mínimos e insuficientes). Sin embargo, la salud indígena y la atención médica institucional siguen siendo precarias en cuanto a recursos humanos y materiales (personal sanitario, medicamentos, etc.), y discriminatorios con relación a la forma y al contenido de la atención que se brinda. En este artículo, detallamos algunas de las intervenciones del gobierno que suponen un avance institucional sobre el tema de interculturalidad en salud pero que, en el fondo, significan la continuidad de políticas arbitrarias y excluyentes.

PALABRAS CLAVES Salud de Poblaciones Indígenas; Discriminación; Racismo; México.

ABSTRACT Over the last 26 years, the Mexican government has developed a number of activities and discourses around what has been called "intercultural health," directed especially at indigenous peoples in Mexico (some 62, according to linguistic criteria). In this way, the government has built health care institutions (rural centers, clinics, and hospitals) in states like Puebla, Nayarit, Oaxaca, Chiapas, Queretaro, and Jalisco, proposing the implementation of cultural pertinence indicators (which are minimal and inadequate). Nevertheless, the health conditions among indigenous populations and the quality of health care provided by public institutions continue to be precarious in terms of human and material resources (health personnel, drugs, etc.) and discriminatory with respect to the form and content of the provided services. This paper describes some of the governmental interventions that purport to be institutional improvements in the field of interculturality, but that actually represent the continuity of arbitrary and exclusive policies.

KEY WORDS Health of Indigenous Peoples; Discrimination; Racism; Mexico. 


\section{INTRODUCCIÓN}

Desde mediados de la década de 1980 hasta la actualidad, en México surgieron novedosas actividades estatales en el campo de la salud indígena, entre ellas, la conformación de asociaciones de terapeutas indígenas; el reconocimiento jurídico a sus actividades curativas; la incorporación en hospitales ubicados en territorios indígenas; la emergencia de oficinas federales y estatales para apoyar las medicinas "tradicionales" y alternativas-complementarias; el inicio, desarrollo e implementación de plataformas teórico-prácticas de cursos, talleres y diplomados dedicados a la denominada "salud intercultural"; cambios y adaptaciones en los establecimientos institucionales que validaran la pertinencia cultural de sus instalaciones; la capacitación de los trabajadores de la salud para adquirir competencias en el terreno intercultural; la enseñanza de la antropología médica y la interculturalidad en escuelas universitarias y centros de enseñanza superior, entre otros aspectos.

Con más de 25 años de experiencias sobre esta política de salud en pueblos originarios de México, es momento de hacer un recuento y elaborar una reflexión sobre sus avances, sus limitaciones y sus posibles adecuaciones. Voces expertas, críticas y reflexivas han surgido a partir de la implementación de los hospitales denominados mixtos, de los gestores interculturales, de los cursos y diplomados y, en general, de la concepción estatal de la salud intercultural ${ }^{(1,2,3,4)}$.

\section{EL INDIGENISMO MEXICANO INTEGRATIVO}

En México, la invasión, conquista y usurpación del territorio por españoles significó poco más de trescientos años de férreo control colonialista y la irrupción de una reproducción combinatoria -biológica y sociocultural- de los pobladores originarios locales con los nuevos residentes foráneos europeos y, más adelante, africanos y asiáticos. El colonialismo externo, y luego el interno, dieron como resultado la invariable subordinación política, social y económica de los pueblos indígenas y descendientes de otros grupos culturales al poder. Gracias al surgimiento de la teoría evolucionista se reconfigura un nuevo panorama, sin embargo, a la par surgió un racismo científico y eurocéntrico desde mediados del siglo XIX, que permitió la identificación de la raza con la nacionalidad, integrando el sentido del mestizo, como base y destino del Ilamado "indio". Bajo la supuesta integración de la denominada "raza cósmica" se realizaron experimentos e intentaron borrar las diferencias étnico-culturales y la evidente desigual distribución de la riqueza. Esta visible desigualdad se reflejaría en el acceso a los servicios educativos y de salud. A mediados del siglo XX surgiría la preocupación estatal por la situación desventajosa de los pueblos originarios, dando origen a las políticas públicas de corte indigenista, es decir, de aquellas acciones dirigidas a los "indios" para ser normalizados y contenidos de alguna manera, al sistema del creciente nacionalismo.

En dicho periodo se institucionalizó la disciplina antropológica (en su visión epistemológica, teórica, metodológica e, incluso, aplicativa) que orientó -en mayor o menor grado- esas políticas públicas dirigidas particularmente a la población originaria.

En América Latina y México, la teoría culturalista o del relativismo cultural influyó en forma notable en la construcción del indigenismo. Sin embargo, su principal expositor Franz Boas presentó propuestas de investigación que chocaban con el culturalismo estadounidense y los intereses de esa nación en el territorio mexicano, sobre todo, en el ámbito de la tradición médico-académica que influyó a la antropología física y que cuestionaba la visión demográfica antirracista y eugenésica dominante ${ }^{(5)}$.

Franz Boas y sus discípulos consideran la inexistencia de culturas superiores e inferiores (planteada por los pensadores evolucionistas) y fundamentan la aplicabilidad de la antropología en función del respeto a 
la existencia de culturas consideradas como diferentes. En oposición a la teoría funcionalista que sirvió a las naciones metropolitanas europeas que buscaban una antropología "interesada en proporcionar herramientas eficientes para el control y buen gobierno de los pueblos sometidos" que conllevara cambios paulatinos, controlados y armónicos de los pueblos por ellos colonizados ${ }^{(6)}$.

Se buscan los posibles vínculos entre el discurso antirracista y el discurso del indigenismo -cómo se auxilian mutuamente y cómo se enlazan en la idea del mestizaje- para señalar la inexistencia de razas puras y la imposibilidad de degeneración de las mezclas. Tal como afirma Molina Enríquez, citado por Bonfil Batalla ${ }^{(7)}$, los mestizos eran los únicos capaces de desarrollar el espíritu nacionalista: los blancos poseían una psicología extranjerizante y los indios una psicología localista. La solución, entonces, estaba en la expansión del mestizaje: los indios deberían convertirse en mestizos, no solo mediante la miscegenación (mezcla de tres troncos raciales en el caso americano de mongoloide, caucasoide y negroide) sino también mediante la castellanización, la educación y el reparto de los latifundios. Solo así se haría efectiva la igualdad legal de la población: observando al mestizo como sinónimo de progreso y al indio como atraso y degeneración racial.

De ahí la necesidad de crear mecanismos para dar este paso crucial, por lo que surgen las acciones integracionistas y asimilacionistas, las que sirvieron de orientación a los antropólogos mexicanos Manuel Gamio, Miguel Othón de Mendizábal, Moisés Sáenz, para promover las líneas teóricas y prácticas del Congreso Indigenista Interamericano celebrado en Pátzcuaro, Michoacán en 1940, que fue fundamental para la creación del Instituto Nacional Indigenista, en 1948.

En ese primer congreso se concluyó que era de interés público reconocer el problema de los pueblos indígenas del continente americano. Y sobre la salud, se recomendaba la instalación en regiones indígenas de "Centros de Medicina Social, Preventiva y Curativa que se ocupen de mejorar las condiciones de salud de los habitantes y de combatir las enfermedades" con especial atención a patologías infectoparasitarias (oncocercosis, paludismo, el mal de pinto, etc.), la creación de escuelas médicas rurales, la investigación científica de la botánica medicinal indígena y el mejoramiento de la alimentación ${ }^{(8)}$.

Gonzalo Aguirre Beltrán fue continuador de la obra de Gamio y actor institucional por excelencia del indigenismo integracionista mexicano. Entre sus textos relativos a la salud, la antropología médica, el curanderismo y la botánica médica, que abarcarían múltiples inquietudes, retos y desafíos teóricoprácticos, se destacan: Programas de salud en la situación intercultural(9), El proceso de aculturación y el cambio socio-cultural en México ${ }^{(10)}$, Medicina y Magia: El proceso de aculturación en la estructura colonial(11) y Antropología Médica: Sus desarrollos teóricos en México ${ }^{(12)}$ que serían la guía y la orientación de las acciones indigenistas en salud durante poco más de cuarenta años ${ }^{(13)}$. Él acuña el concepto y genera formas de intervención de tipo aculturativo en las que hacía hincapié en que "[el] fin del indigenismo mexicano no es el indígena, es el mexicano", que tendrá que integrarse a la nación como ciudadano producto del indigenismo. El mestizo es "instrumento unificador de la heterogeneidad nacional...", sin embargo, surge conflicto por la explotación y dominación de este hacia el indio, lo cual trata de revertir a través de la "integración intercultural" fundada en la "aculturación planificada", definida como el proceso social de cambio inducido que es adoptado y apropiado por individuos y grupos sociales, y que facilita la asimilación e incorporación de las personas al sistema económico y político capitalista. El Instituto Nacional Indigenista (INI) absorbió la propuesta de Aguirre Beltrán sobre la necesidad de generar un programa de salud acorde con la situación intercultural del país ${ }^{(4)}$. Bonfil Batalla ${ }^{(14)}$ refería esto como la construcción de un terreno común de solidaridad bajo el sentido de legitimidad tradicional y no solo de imposición legal del sistema oficial, lo que daría paso a una "intervención culturalmente apropiada para la medicina oficial". 


\section{EL INDIGENISMO MEXICANO PARTICIPATIVO}

El INI adoptó una política de creciente penetración de la biomedicina en el ámbito indígena, con muy escasa relación directa con los terapeutas tradicionales. Durante el gobierno de Carlos Salinas (1988-1994) -que se caracterizó por el impulso de una política económica de corte liberal, con privatización de paraestatales y disminución en la intervención del Estado-, con el antropólogo Arturo Warman en la dirección y Carlos Zolla como responsable de asuntos de salud, se inicia una nueva etapa participativa en la relación con los médicos indígenas: se logran estructurar alrededor de 60 organizaciones regionales, se realizan dos congresos nacionales, se crea la Biblioteca de la Medicina Tradicional Mexicana con activa participación indígena, y se modifica en 1990 el único hospital del INI en el país, ubicado en Cuetzalan (Puebla), para transformarlo en un centro híbrido de atención, donde además de la atención biomédica se ofrecía, intramuros, el servicio de diversos terapeutas indígenas pertenecientes a la organización de médicos indígenas de la Sierra Norte de Puebla ${ }^{(15,16)}$. En 1990, es traspasado a la Secretaría de Salud estatal, y se replica en otras unidades hospitalarias de Puebla y fuera de ella en Jesús María, en el estado de Nayarit.

Según su creador, este cambio obedeció a la conjunción de cuatro ejes rectores: a) la existencia en la comunidades indígenas de un "sistema real de salud" en el que aparece la biomedicina, la medicina casera o doméstica y la medicina tradicional, b) la aceptación de la estrategia de atención primaria de salud desarrollada por la Organización Mundial de la Salud (OMS), c) la implementación de los sistemas locales de salud, promovidos por la Organización Panamericana de la Salud (OPS), y d) el reconocimiento completo a las actividades de los médicos indígenas en sus zonas de residencia ${ }^{(15)}$.

Duranteel gobierno de VicenteFox (20002006), el INI se transforma (y, en realidad, se degrada) en Comisión para el Desarrollo de los Pueblos Indígenas (CDI), con una política de continuidad asistencialista.

Con el fin de cumplir las metas para el desarrollo de los pueblos indígenas en el contexto del Plan Puebla-Panamá, en el año 2001 se crea el área de Planeación Estratégica y Desarrollo Regional y la Coordinación General del Plan Puebla-Panamá (ahora Proyecto Mesoamérica). Se genera la comisión Sur-Sureste de México a cargo de los estados de Puebla, Veracruz, Tabasco, Guerrero, Oaxaca, Chiapas, Campeche, Yucatán y Quintana Roo, regiones en donde se concentra la mayoría de la población indígena del país. Puebla, en su carácter de estado miembro de dicha comisión, instaura los Hospitales Integrales con Medicina Tradicional. Además, a nivel federal, se crea la Coordinación de Salud de los Pueblos Indígenas, que posteriormente cambia de denominación y conforma la Dirección de Medicina Tradicional y Desarrollo Intercultural (DMTDI) en la Secretaría de Salud, cuyo eje de desarrollo se perfilaría hacia la conformación de programas mixtos de atención y posteriormente se reorientó hacia la visión intercultural( ${ }^{(4)}$.

En este periodo se dejan de apoyar económica e intelectualmente los planes específicos de medicina tradicional. Sin embargo, en el año 2006, el gobierno federal lleva a cabo la modificación de varios artículos de la Ley General de Salud ${ }^{(17)}$, y se incorpora la "medicina tradicional indígena" en dicho marco legal que en su artículo 6 fracción VI bis indica "...promover el conocimiento y desarrollo de la medicina tradicional indígena y su práctica en condiciones adecuadas" y en su artículo 93 establece:

De la misma manera reconocerá, respetará y promoverá el desarrollo de la medicina tradicional indígena. Los programas de prestación de la salud, de atención primaria que se desarrollan en comunidades indígenas, deberán adaptarse a su estructura social y administrativa, así como su concepción de la salud y de la relación del paciente con el médico, respetando siempre sus derechos humanos. ${ }^{(17)}$ 


\section{EL INDIGENISMO MEXICANO AL INICIO DEL SIGLO XXI}

La DMTDI de la Secretaría de Salud federal tiene un protagonismo más discursivo y mediático que pragmático ${ }^{(4,18,19,20,21,22,23,24)}$, en gran parte explicado por la ausencia de un presupuesto asignado, de personal suficiente y de contactos reales con los pueblos indígenas. No obstante, tiene visibilidad en múltiples cursos de capacitación y "sensibilización", inicialmente auxiliado por una organización no gubernamental (ONG) dirigida por un grupo de antropólogos universitarios que fueron contratados por la fugaz y desaparecida Coordinación Federal dedicada a la salud y nutrición de pueblos indígenas $(2001-2004)^{(18)}$

Posteriormente, la DMTDI brinda cursos virtuales por Internet $y$, en menor medida, presenciales, elabora un libro sobre salud intercultural, varios folletos dedicados a programas específicos (por ejemplo, de salud reproductiva y relación médico-paciente), se aboca a establecer algunas recomendaciones en centros de atención médica, y dedica esfuerzos a la implantación del parto vertical en varios hospitales del país (Secretaría de la Defensa Nacional, Hidalgo, San Luis Potosí, entre otros). Su presencia se hace visible en algunos estados de la República, en especial, en el centro y sureste del país, donde las secretarías estatales establecen dependencias dedicadas a la atención relacionada con la medicina tradicional, las medicinas complementarias y la interculturalidad: Querétaro, Hidalgo, Puebla, Veracruz. Sin embargo, se observa una precaria influencia en los estados de mayor presencia indígena como Michoacán, Guerrero, Oaxaca y Chiapas.

En Querétaro, el Programa de Interculturalidad y Medicina Tradicional con limitaciones ideológicas y presupuestarias, realiza capacitación al personal de salud y en 2013 establece en Amealco -región con casi un $57 \%$ de población ña 'ñú (otomí)- un centro de salud con hospitalización que añade un área de medicina tradicional que brinda consulta curanderil (curandero, huesero, hierbero y partera), temazcal, atención de parto y farmacia herbolaria. Se establecen normas de uso y para la capacitación del personal de salud se emplearon técnicas audiovisuales de cortometraje (filmación de historias de vida de curanderos) para demostrar su existencia y vigencia curativa ${ }^{(25)}$. Se dice que los terapeutas seleccionados constituyen personal "voluntario" y se les ofrece una pequeña compensación mensual (6.500 pesos mensuales). Aún no se los reconoce como "unidad de salud culturalmente competente" por carecer de algunos indicadores, entre los cuales se destaca la ausencia de señalética en idioma indígena local. Cabe mencionar que los criterios de acreditación de las "Unidades de Salud Culturalmente Competentes" son: contar con un $60 \%$ de personal capacitado con perspectiva intercultural; contar con promotor de salud bilingüe intercultural; integrar a los médicos indígenas en "comunidades de diálogo"; difundir información sobre "cosmogonía local" y señalética en lengua indígena; favorecer actitudes de respeto, comprensión y amabilidad del personal de salud con los usuarios, Ilamándolos por su nombre sin utilizar diminutivos ${ }^{(26)}$. Obsérvese que no se incluye el parto vertical en tales establecimientos de salud. Finalmente, se encuentra en proceso un módulo semejante en Tolimán, otra región habitada por mayoría indígena ñáñú.

En Hidalgo, se capacita al personal del hospital civil ubicado en Pachuca (alrededor de 1.000 empleados), se crea un módulo de atención en prácticas complementarias (acupuntura, naturismo y algo de medicina popular), se atienden en forma no oficial unos escasos partos verticales (no reconocidos en las estadísticas porque el jefe del servicio de gíneco-obstetricia se opone a esta práctica por considerarla inadecuada y sin recursos materiales para su ejecución), solo dos o tres médicos responden al Ilamado intercultural y la Secretaría de Salud federal certifica al hospital como unidad "culturalmente competente" junto con los ubicados en Huejutla, Ixmiquilpan y Tula. Los dos primeros con presencia de traductores indígenas (sin categoría laboral específica) y el último, al 
parecer, convertido en el centro nacional de aprendizaje de atención del parto vertical. (RG, comunicación personal). Cabe mencionar que del primero de estos hospitales surgió la Ilamada "Lady Huejutla", una estudiante de medicina de la Universidad Autónoma del Estado de Hidalgo, quien en las redes sociales señaló su gozo de retornar a la "civilización" (Pachuca) después de haber estado con esos "aborígenes" que hablan el náhuatl, hecho que impedía una adecuada comunicación con los pacientes de una comunidad cercana a la cabecera municipal. El asunto mereció su baja del servicio social, una amonestación de la Comisión de Derechos Humanos del Estado de Hidalgo con recomendación de "orientación profesional" y su envío a un hospital de Jalisco(27).

En Veracruz, se crea formalmente en el 2006 el Programa de Salud Indígena y Medicina Tradicional con Enfoque Intercultural, y realiza talleres formativos para el personal de salud (el $64 \%$ biomédicos y el $15 \%$ enfermeras) y otros dirigidos a la conformación de "mediadores interculturales", cuya función es buscar espacios de diálogo con los médicos indígenas. A fines de ese año, realizan un encuentro con 115 médicos indígenas del norte de Veracruz (Poza Rica, Pánuco y Tuxpan) donde los indígenas solicitan reconocimiento y trato respetuoso e igualitario, en tanto que el gobierno estatal ofrece continuar con el apoyo a la práctica de la medicina indígena en todo el territorio veracruzano ${ }^{(28)}$. Posteriormente, se crea una Coordinadora Estatal del Programa de Interculturalidad dependiente del Departamento de Salud del Migrante y Pueblos Indígenas que diseña, en el año 2013, un proyecto de Modelo de Salud Indígena con Pertinencia Cultural cuyos principales objetivos -basados en el derecho nacional e internacional- son los de capacitar al personal de salud en el enfoque intercultural y de género posicionando el uso de las lenguas indígenas, fortalecer la medicina tradicional, realizar talleres comunitarios interculturales de promoción de salud y adaptar las unidades de atención médica a la "cultura de los usuarios" de comunidades indígenas ${ }^{(29)}$.
En términos operativos se mencionan avances en la capacitación a funcionarios jurisdiccionales y de unidades médicas; la contratación efectiva de quince promotores de salud bilingües interculturales, egresados de la Universidad Veracruzana Intercultural (UVI); la enseñanza de idioma náhuatl en Orizaba y Río Blanco; y los centros de salud culturalmente competentes, uno de ellos en Filomeno Mata, comunidad totonaca de la Sierra Madre Oriental, el primero de los 140 que están proyectados en regiones indígenas $^{(26)}$. Cabe resaltar que la contratación, el reconocimiento y el trato laboral han sido en calidad de "promotores de salud" y no como licenciados egresados en la carrera universitaria de gestión intercultural (información proporcionada en abril de 2015 por A. Isunza, ex responsable de la vinculación de la UVI con la Secretaría de Salud de Veracruz).

En un reciente estudio sobre derechos humanos y salud intercultural en el hospital de Tonalapan, municipio de Mecayapan, Veracruz, considerado como "hospital intercultural" que atiende población nahua y popoluca, se encontró que la interculturalidad se reduce únicamente al uso de señalética, que no existe capacitación específica del personal de salud, que hubo en el pasado una licenciada de gestión intercultural como traductora pero que fungía de multiusos (mandadera), y la relación con las parteras es subordinada e instrumental, dado que no se les permite atender partos a pesar de la capacitación ofrecida por la propia secretaría de salud. Se concluye que:

...el sistema médico hegemónico no respeta particularidades e individualidades del modelo subordinado, sino que impone, avasalla y no concerta. [...] las políticas interculturales en salud; así como la implementación de dichas políticas en el hospital de Tonalapan, obstaculizan la realización del derecho a la salud de los indígenas. ${ }^{(30)}$

En Puebla, se continúa con el proyecto de los hospitales mixtos. Se construyó un nuevo hospital general en Cuetzalan en la periferia 
de la población y se han replicado hasta 14 hospitales más denominados "integrales con medicina tradicional" en diversas regiones indígenas del estado. Múltiples investigaciones con fines evaluativos se han realizado, algunos conocidos en las redes sociales y otros en proceso. En ellos se constatan avances, estancamientos y conflictos. La interculturalidad ha sido centrada exclusivamente en la presencia de los módulos de medicina tradicional en los espacios biomédicos con la notoria subordinación de los terapeutas tradicionales ${ }^{(31,32,33)}$. En la página electrónica de la Secretaría de Salud del estado de Puebla, se mantiene desactivada la información sobre los hospitales integrales que cuentan con módulo de medicina tradicional. Entonces, como señalan Duarte et al.:

El hospital de Cuetzalan y el proyecto de Hospitales Integrales con Medicina Tradicional constituyen un avance y un intento plausible por cumplir con algunos de los compromisos del Convenio 169 de la OIT y de las recomendaciones de la OPS/OMS, pero siguen siendo planeados, ejecutados y evaluados "desde arriba" por las instituciones gubernamentales, en procesos en los que las comunidades no tienen poder de decisión y apenas son consultadas. ${ }^{(31)}$

El único estado de la República que decidió imitar la experiencia del hospital mixto poblano fue Nayarit. En la comunidad cora de Jesús María se construyó el hospital que contiene atención biomédica con una sección dedicada a la medicina tradicional. El personal de salud realiza rotación cada 15 días y la relación con los terapeutas tradicionales es mínima. Todos los partos son atendidos por los médicos (en la forma convencional sin el uso de mobiliario para el parto vertical recomendado por el nivel federal). Todos los días se encuentra una partera indígena que únicamente se dedica a la atención de las mujeres embarazadas que solicitan el servicio. Además de curandero marakame, hay hierberos y se observa una enorme precariedad en los recursos materiales del módulo.
En el estado de Oaxaca la penetración del programa federal es pobre, se continúan acciones en algunos centros de atención médica tradicional (como Capulalpam de Méndez), aplicación de un modelo de parto "intercultural" en Tlalixtac de Cabrera y un ayuntamiento que ha implementado medidas para que la atención obstétrica sea mejorada, es el caso de Santa María Tlahuitoltepec que indica tratar con respeto y dignidad a las embarazadas y sus familiares ${ }^{(34)}$.

Por otra parte, se destacan las acciones de agrupaciones académicas y ONG que tienen como meta la mejora de la atención intercultural del embarazo/parto/puerperio y la disminución de la mortalidad materna en el estado. Una asertiva evaluación de los servicios médicos gubernamentales en Oaxaca demuestra que los centros de salud suelen atender con relativa satisfacción la fase prenatal, pero derivan a hospitales la atención del parto, sin importar si son normales o complicados, y el personal de salud no aplica competencias en el terreno intercultural. Los autores recomiendan la divulgación en el sector salud del marco normativo y los derechos relacionados con la salud materna, reforzar las competencias técnicas obstétricas e interculturales de todo el personal de salud, lograr un suficiente abasto de equipos, insumos y medicamentos, y establecer que los hospitales se dediquen a la atención de partos complicados y los centros de salud puedan atender los partos normales con la calidad anotada en la normatividad institucional ${ }^{(35)}$.

Además, los estudios antropológicos recientes en Oaxaca demuestran la enorme desigualdad social y discriminación estructural de las mujeres indígenas ante la presencia de complicaciones durante el embarazo/ parto/puerperio, como lo señala Paola Sesia:

Son los municipios indígenas los que tienen las peores carreteras, los que están menos comunicados y más distantes de los servicios médicos en Oaxaca. Son las familias indígenas de estos municipios de extrema marginación las que no tienen posibilidad real de acceder a servicios médicos, aun así esta exclusión es 
profundamente violatoria de las leyes y normas federales y estatales que garantizan en papel el derecho a la salud. ${ }^{(36)}$

En el estado de Chiapas, el hecho más sobresaliente es la construcción en los Altos de un hospital inaugurado en mayo de 2010 que, por tener una sección de medicina tradicional, es denominado como "Hospital de las Culturas". Además de incompleto, resulta el mejor ejemplo de una unidad médica hospitalaria construida a espaldas de las comunidades indígenas contraviniendo la consulta previa que mandata el Convenio 169 de la Organización Internacional del Trabajo (OIT). Se construyó - parcialmente- encima de un panteón (por ello ahora es conocido por el personal de salud como el "Hospital de las sepulturas") y pese a tener un amplio espacio para la atención del parto "vertical" su uso ha sido nulo, pues no llegan parturientas porque la mayoría de ellas son canalizadas al actual Hospital de la Mujer (antes Hospital Regional), que se encuentra en el centro de San Cristóbal de Las Casas. Posee también un temazcal que no se usa por fallas de ingeniería y las parteras son capacitadas (y controladas) por las autoridades sanitarias locales con escaso respeto a usos y costumbres en su práctica obstétrica.

De los dos nosocomios ubicados en San Cristóbal se tienen experiencias negativas en la atención de pacientes indígenas que demuestran que el racismo y la discriminación siguen siendo problemas no resueltos, pese a los discursos interculturales esgrimidos por los funcionarios de salud del estado ${ }^{(37,38)}$.

En Guerrero, los esfuerzos federales tampoco han prosperado en las políticas públicas estatales. Son las ONG quienes están desarrollando un mayor esfuerzo para lograr reducir la mortalidad materna que es la más elevada del país ${ }^{(39)}$.

En el caso de Colima, a partir de 2007, se realizaron esfuerzos para incluir acciones en favor de la medicina tradicional en el primer nivel de atención, en la comunidad de Suchitlán, Comala, dialogando con el personal de salud sobre la necesidad de la interculturalidad y la construcción de un temazcal, acciones que no prosperaron. Posteriormente, se contrató una partera profesional de la escuela de San Miguel de Allende, Guanajuato, en el Hospital de la Mujer, para cumplir con la política pública que demandaba la DMTDI, la cual continúa laborando ${ }^{(4)}$.

En la región huichola de Jalisco recién se acaba de inaugurar, en Huejuquilla, El Alto, el denominado Hospital Comunitario Multicultural, donde se cuentan con los servicios biomédicos habituales más la presencia de terapeutas indígenas ${ }^{(40)}$. En una visita reciente se observó que aún no funcionaba la hospitalización (pese a que ya han pasado dos meses desde su apertura debido a problemas de insumos). Labora un entusiasta traductor que coordina el servicio de medicina tradicional donde los diversos especialistas (partera, sobador, huesero, hierbero y marakame) tienen presencia rotativa por acuerdo con las asambleas comunitarias. Hasta ahora están asistiendo más pacientes mestizos, y se dice que no se permitirá la atención indígena del parto por lo que la partera se concretará sólo a realizar controles de embarazo. Finalmente, es nula la capacitación intercultural del personal de salud (diario de campo, 2016).

Por otra parte, el Instituto Mexicano del Seguro Social (IMSS) antes IMSS-Oportunidades y ahora IMSS-Prospera, de su antiguo programa de interrelación con la medicina tradicional solo ha priorizado el diálogo con las parteras con fines instrumentales, se relaciona de manera parcial a las normas de la DMTDI, tiene una oficina de Apoyo a la Atención a Pueblos Indígenas que intenta, desde hace un año, diseñar e implementar un modelo de atención intercultural en dos o tres hospitales con población indígena mayoritaria, que incluya la presencia de mediadores interculturales, y de espacios para la atención del parto en condiciones interculturales. Sin embargo, el proceso -a pesar de las buenas intenciones- continúa paralizado.

Para resumir, durante medio siglo el gobierno mexicano efectuó acciones indigenistas de carácter integracionista, pero a partir de los años noventa del siglo pasado se iniciaron otras políticas públicas de indigenismo 
participativo, que dieron origen a diversos y novedosos ensayos que se les empezaron a calificar de "interculturales".

Sin embargo, desde una perspectiva crítica en esta etapa aparecieron -en forma cada vez creciente- los estudios socioantropológicos que muestran el carácter discriminatorio y excluyente de las políticas públicas hacia los pueblos indígenas, asiáticos y afrodescendientes, que expresan la enorme desigualdad social a la que se enfrentan y que, en el plano de la atención biomédica, existen barreras estructurales (económico-políticas) y no tanto culturales, que impiden un acceso real a los servicios de salud ${ }^{(3)}$.

\section{ALGUNAS CONSIDERACIONES}

Con la breve descripción de las actividades estatales sobre salud indígena en México, nos podemos percatar que las acciones son parciales, limitadas e insuficientes para atender de manera adecuada (en términos cuantitativos y de calidad) a los pueblos originarios, que constituyen por lo menos de un $10 \%$ a un $15 \%$ de la población mexicana.

La pertinencia de los servicios de salud -en términos interculturales- es aún muy precaria, con múltiples contradicciones y ambigüedades en los planes, programas y estrategias institucionales. Por si fuera poco, entre el 2015 y mediados del 2016, el gobierno de Peña Nieto ha realizado ajustes a la meta de crecimiento de la economía mexicana, por lo que ha recortado el gasto público, donde el rubro de salud ha sido uno de los principales afectados ${ }^{(41)}$. Ello se verá reflejado en menores recursos destinados a programas, hospitales y centros de investigación. Existe una fundamentación jurídica en los niveles internacionales (Convenio 169 de la OIT, firmado y ratificado por México en 1990), nacionales (Constitución Política y Ley General de Salud) y estatales, sin embargo, el incumplimiento es la norma. No se observan verdaderos apoyos políticos y financieros a los proyectos de la medicina tradicional mexicana, lo cual es un claro reflejo de la hegemonía de la biomedicina en el aparato gubernamental (tanto federal, estatal como municipal).

Históricamente, el IMSS mantuvo un proyecto de interrelación positiva con los terapeutas tradicionales en la década de los ochenta del siglo pasado que ahora está en plena declinación (con excepción de la relación -también restringida- con las parteras por razones de manejo político en programas de planificación familiar).

En la actualidad, la Secretaría de Salud y su DMTDI es la dependencia federal que se responsabiliza, de forma incompleta, de la salud indígena, con muy escaso contacto y presencia con los pueblos originarios. Permea más el discurso y la presunción nacional e internacional. Por ejemplo, la señora Margarita Zavala, esposa del expresidente Felipe Calderón, en visita oficial a Margaret Chan, directora de la Organización Mundial de la Salud (OMS) en Ginebra, Suiza, presumió en febrero de 2012 -y así lo consignaron los periódicos del momento- que el gobierno mexicano estaba brindando un nítido apoyo al tema de la interculturalidad en servicios médicos:

\begin{abstract}
Margarita Zavala subrayó el impulso a un enfoque intercultural en el Sistema Nacional de Salud y la creación de centros de medicina tradicional en estados como Puebla y Chiapas, entre otros, como complemento al trabajo que realizan las unidades médicas, con el fin de asegurar la atención a la población indígena, principalmente. ${ }^{(42)}$
\end{abstract}

$\mathrm{Y}$, sobre todo, influye el grado de inserción en las plataformas sanitarias institucionales preexistentes, donde las políticas públicas interculturales se inscriben y se desvanecen en una fragmentada e inequitativa estructura que genera:

...responsabilidades paralelas, traslapadas y a menudo conflictivas entre distintos actores político-burocráticos, mismas que entrañan, además de dificultades en la comunicación y administración, fuertes inequidades en la distribución de los 
recursos destinados a distintos sectores de la población. ${ }^{(43)}$

Esta fragmentación e inequidad se refleja en las decisiones y omisiones en los distintos niveles gubernamentales de atención sanitaria. Así, por ejemplo, hay aproximaciones y distanciamientos entre la Secretaría de Salud federal (que apoya al Seguro Popular) y el Programa IMSS-Prospera, cada uno con sus propios modelos de acción médica-comunitaria (y sus diferentes recursos) en poblaciones indígenas del país.

El impacto de las políticas interculturales aun es desconocido e incierto. Parte del desconocimiento se debe a la ausencia de estadísticas oficiales con diferenciación étnica. Recién a partir de 2014 se interroga en el Hospital General de México "Eduardo Liceaga" sobre el origen indígena de sus pacientes hospitalizados. Los datos iniciales apuntan hacia un promedio del $5 \%$ de pacientes indígenas en los ingresos del hospital en 2014 $4^{(44)}$, con probable subregistro al considerar solo el criterio lingüístico sin incluir la autoadscripción. En estudios epidemiológicos puntuales y rigurosos en regiones y microrregiones sobre mortalidad y morbilidad diferenciada por etnias se podrán observar los cambios e impactos de las políticas interculturales en salud, con mejores posibilidades de hacer una relación de causa/efecto.

Los servicios de salud en pueblos originarios siguen siendo calificados como ausentes $y$, si existe, es calificado como nefasto e irregular. Durante 2015, en Simojovel, Chiapas, región indígena tzotzil, se presentaron 2 muertes y 29 afectados por vacunación contra hepatitis $B$, tuberculosis y rotavirus, la investigación de las causas fue por mal manejo del material. El agente municipal Higinio Pérez López se quejó con amargura:

No sólo no hay médicos; tampoco hay medicamentos ni material de curación. Y cuando hay médicos, no hablan nuestra lengua y se nos dificulta explicar nuestras dolencias en castilla. Y en muchas ocasiones sufrimos maltrato físico y verbal del personal médico por ser indígenas. ${ }^{(45)}$
Y en la capital de Oaxaca, hubo un paro de actividades médicas y administrativas en el principal hospital público, en el que los médicos exigieron que haya una necesaria ampliación del servicio de gineco-obstetricia dado que resulta completamente insuficiente para la atención obstétrica de las pacientes ${ }^{(46)}$. Además, es un absurdo institucional que todo parto sea atendido en el segundo nivel de atención, es decir, en hospitales, y no se mejore la red de establecimientos de salud rurales y suburbanos, en la que también se han informado múltiples partos mal atendidos.

Con respecto al papel de la antropología en los procesos de s/e/a, es relevante su enfoque cualitativo para dar cuenta de los progresos y las limitaciones de las políticas públicas, entendidas no solo como acciones de gobierno, sino también de participación de los conjuntos sociales, y de cuáles son las necesidades y demandas de un buen servicio en los establecimientos institucionales de salud, y su mejor orientación en los pueblos sean estos originarios o no.

Los avances desde la antropología médica crítica y la epidemiología sociocultural orientan hacia una mejor comprensión de los fenómenos de s/e/a donde se entienden las limitaciones estructurales (y desigualdades sociales) de la atención biomédica, así como el auténtico papel de las medicinas tradicionales y la autoatención en las comunidades rurales y urbanas. Diversos investigadores, algunos provenientes de los mismos pueblos indígenas, han asumido una postura crítica de las actuales políticas interculturales pues a pesar de expresar un discurso en el que se resaltan el respeto a la cultura y la participación activa de las comunidades indígenas, en la acción se confirman formas pragmáticas de exclusión ${ }^{(47,48,49)}$. El enfoque crítico de la interculturalidad en salud, entre otros aspectos, señala que el discurso intercultural, al resaltar el aspecto simbólico-cosmogónico de las terapéuticas indígenas, tiende a anular el contexto sociocultural en el que se expresan, tales como el proceso de empobrecimiento; el despojo del territorio, la discriminación con base en imaginarios racistas, así como el poco o nulo acceso a bienes y servicios. 
A la par, existe una amplia diversificación en la utilización del concepto de interculturalidad ya sea como enfoque, herramienta metodológica, política pública y de certificación. En esta época, algunas poblaciones indígenas en México y América Latina han asumido la interculturalidad como discurso de utilidad para ser identificadas y potencializar transacciones, ya que el esquema mediante el cual están planteadas las políticas de interculturalidad en salud no permiten el diálogo entre conocimientos de las comunidades y pueblos para establecer las formas de participación por lo que se manifiestan comportamientos polarizados a favor y en contra, en torno a la asimilación y la resistencia ${ }^{(50)}$.

Con todo lo expuesto, necesitamos hoy más que nunca de un diálogo formal, flexible y constructivo en la incorporación de la antropología en salud -y otras disciplinasen los problemas contemporáneos de salud, tanto de pueblos indígenas, afrodescendientes y grupos asiáticos, como de la ciudadanía en general.

\section{REFERENCIAS BIBLIOGRÁFICAS}

1. Lerín S. Interculturalidad y salud: recursos adecuados para la población indígena o propuestas orientadas a opacar la desigualdad social [Internet]. 2005 [citado 26 may 2016]. Disponible en: https://goo.gl/RrkEt7.

2. Menéndez E. Interculturalidad, "diferencias" y antropología at home: Algunas cuestiones metodológicas. In: Fernández G. (ed.) Salud e interculturalidad en América Latina: Antropología de la salud y crítica intercultural. Quito: Ediciones Abya-Yala; 2006.

3. Haro A. Interculturalidad en salud, viejos pretextos, nuevos desafíos. In: Ramírez-Hita S. Salud intercultural: Crítica y problematización a partir del contexto boliviano. La Paz: Instituto Superior Económico Andino de Teología; 2011.

4. Peña EY, Hernández-Albarrán L. Entre saberes ancestrales y conocimientos contemporáneos: Las representaciones y prácticas curativas en Suchitlán, Comala, Colima. México: Instituto Nacional de Antropología e Historia; 2013.

5. Rutsch M, Entre Nicolás León y Franz Boas: Una disputa y sus consecuencias en la Antropología Física de México. La Habana: VII Conferencia Internacional Antropología; 2004.

6. Díaz-Polanco H. Las teorías antropológicas: El evolucionismo. México: Editorial Línea; 1983.

7. Bonfil Batalla G. Andrés Molina Enríquez y la Sociedad Indianista Mexicana: El indigenismo en vísperas de la Revolución. Anales del Instituto Nacional de Antropología e Historia.1967;(XVIII):217-232.
8. Val J, Zolla C. Documentos fundamentales del indigenismo en México. México: UNAM, Programa Universitario de Estudios de la Diversidad Cultural y la Interculturalidad; 2015.

9. Aguirre-Beltrán G. Programas de salud en la situación intercultural. México: Instituto Indigenista Interamericano; 1955.

10. Aguirre-Beltrán G. El proceso de aculturación y el cambio sociocultural en México. México: Universidad Nacional Autónoma de México; 1957.

11. Aguirre-Beltrán G. Medicina y magia: El proceso de aculturación en la estructura colonial. México: Instituto Nacional Indigenista; 1963.

12. Aguirre-Beltrán G. Antropología médica: Sus desarrollos teóricos en México. México: Centro de Investigaciones y Estudios Superiores en Antropología Social; 1986.

13. Zolla C. Antropología médica, salud y medicina en la obra de Gonzalo Aguirre Beltrán. In: Baez J. (coord.). Gonzalo Aguirre Beltrán: Memorial crítico. Xalapa: Editora del Gobierno del estado de Veracruz; 2008.

14. Bonfil Batalla G. Hacia nuevos modelos de relaciones interculturales. México: Consejo $\mathrm{Na}$ cional para la Cultura y las Artes; 1993.

15. Zolla C. Del IMSS-Coplamar a la experiencia del Hospital Mixto de Cuetzalan: Diálogos, asimetrías e interculturalidad médica. In: Argueta, Corona-M, Hersch (coords.). Saberes colectivos y diálogo de saberes en México. Cuernavaca: UNAM, INAH, Universidad Iberoamericana; 2011. 
16. Argueta Villamar A, Zolla C, Mata S, García I, García I, Becerra R, Pérez G, Altbach D, Martínez A. La medicina tradicional indígena en México: el largo camino para su legalización y reconocimiento. In: Argueta Villamar A, Gómez Salazar M, Navia Antezana J. (coords.). Conocimiento tradicional, innovación y reapropiación social. México: Siglo XXI Editores, UNAM; 2012.

17. Estados Unidos Mexicanos. Decreto que reforma y adiciona diversas disposiciones de la Ley General de Salud. Diario Oficial de la Federación. 2006;DCXXXVI(13):77-78.

18. Secretaría de Salud, Coordinación de Salud para Pueblos Indígenas. Salud y nutrición para población marginada rural e indígena [Internet]. [citado 26 may 2016]. Disponible en: https://goo. $\mathrm{gl} / \mathrm{XKs} 3 \mathrm{WN}$.

19. Secretaría de Salud. Fortalecimiento y desarrollo de la medicina tradicional mexicana y su relación intercultural con la medicina institucional [Internet]. 2004 [citado 26 may 2016]. Disponible en: https://goo.gl/EhZRuW.

20. Secretaría de Salud. Programa de Acción Específico: Interculturalidad en Salud, Mejora de acceso a medicamentos (2007-2012). México: Secretaría de Salud; 2007.

21. Secretaría de Salud. Interculturalidad en Salud: Experiencias y aportes para el fortalecimiento de los servicios de salud. México: Secretaría de Salud; 2008.

22. Secretaría de Salud. Programa de Acción Específico 2007-2012: Medicina tradicional y sistemas complementarios de atención a la salud. México: Secretaría de Salud; 2008.

23. Secretaría de Salud. Programa de Acción Específico 2007-2012: Interculturalidad en salud. México: Secretaría de Salud; 2008.

24. Secretaría de Salud. Metodología intercultural para la realización de consultas informadas [Internet]. México: Subsecretaria de Innovación y Calidad, Dirección de Medicina Tradicional y Desarrollo Intercultural [citado 26 may 2016]. Disponible en: https://goo.gl/bZmnTo.

25. Arciga L. El cortometraje y su utilidad metodológica en procesos de investigación de salud intercultural: el caso del Centro de Salud con Hospitalización de Amealco, Querétaro. [Tesis de licenciatura en Sociología]. Santiago de Querétaro: Facultad de Ciencias Políticas y Sociales. Universidad Autónoma de Querétaro; 2013.

26. Secretaría de Salud del Estado de Veracruz. Modelo de salud indígena con perspectiva inter- cultural [Internet]. 2014 [citado 28 may 2016]. Disponible en: https://goo.gl/araLVe.

27. García I. Surge lady huejutla en Hidalgo, estudiante de medicina que discriminó a indígenas [Internet]. 2015 [citado 26 may 2016]. Disponible en: https://goo.gl/yGmXGR.

28. Rodríguez-Hernández A, Barrera I, VázquezAguilar K, Cortés E. Medicina tradicional en la Huasteca y el Totonacapan: Socialización de una experiencia de intervención institucional con enfoque intercultural en el Estado de Veracruz. México: Secretaría de Salud, Programa de Salud Indígena y Medicina Tradicional con Enfoque Intercultural, Xalapa; 2008.

29. Secretaría de Salud del Estado de Veracruz. Componente salud indígena [Internet]. 2015 [citado may 2016]. Disponible en: https://goo.gl/ HYZULA.

30. Isunza A. Derecho a la salud: políticas y prácticas de la salud intercultural: Estudio de caso con enfoque relacional en el hospital comunitario de Tonalapan, municipio de Mecayapan, Veracruz, México. [Tesis de doctorado en Ciencias en Salud Colectiva]. México: Universidad Autónoma Metropolitana; 2015.

31. Duarte MB, Brachet-Márquez V, Campos-Navarro $R$, Nigenda G. Políticas nacionales de salud y decisiones locales en México: el caso del Hospital Mixto de Cuetzalan, Puebla. Salud Pública de México. 2004;46(5):388-398.

32. Rohrbach-Viadas C. Comparemos medicinas: Cinco hospitales integrales. Puebla: Secretaría de Salud de Puebla; 2005.

33. Benguigui J. L'intégration politique d'une pratique culturelle de santé: le cas des sages-femmes traditionnelles dans les hôpitaux intégraux avec médecine traditionnelle de Puebla, Mexique. Sorbonne, Paris: Université Paris Descartes. Faculté des Sciences Humaines et Sociales; 2014.

34. Sesia P. (ed.). Adecuación intercultural de servicios de salud materna en América Latina: Lecciones aprendidas y retos pendientes. México: Centro de Investigaciones y Estudios Superiores en Antropología Social, Comité Promotor por una Maternidad Segura, Family Care International; 2013.

35. Sachse M, Sesia P, Pintado A, Lastra Z. Calidad de la atención obstétrica desde la perspectiva de derechos, equidad e interculturalidad en centros de salud en Oaxaca. Revista CONAMED. 2012;17(Supl 1):S4-S15. 
36. Sesia P. El papel de la desigualdad social en la muerte de mujeres indígenas oaxaqueñas durante la maternidad: Aportes desde una epidemiología social y una antropología médica "critica". In: Haro A. (coord.). Epidemiología sociocultural: Un diálogo en torno a su sentido, método y alcances. Buenos Aires: Lugar Editorial; 2011.

37. Ruiz-Llanos A. Derechos humanos, ética e interculturalidad. In: Campos-Navarro R. [coord.] Antropología médica e interculturalidad. México: Facultad de Medicina / Programa Universitario de Estudios de la Diversidad Cultural y la Interculturalidad, McGraw-Hill Interamericana; 2016.

38. De las Heras J, Campos-Navarro R. Los hospitales "interculturales" en México: Los casos de Cuetzalan (Puebla) y San Cristóbal de Las Casas (Chiapas). España: inédito; 2016.

39. Berrio LC. Vigilancia y diálogo social: El papel de las organizaciones de la sociedad civil en el diseño, implementación y monitoreo de servicios de salud materna con pertinencia intercultural. In: Sesia P. (ed.). Adecuación intercultural de servicios de salud materna en América Latina: Lecciones aprendidas y retos pendientes. México: Centro de Investigaciones y Estudios Superiores en Antropología Social, Comité Promotor por una Maternidad Segura, Family Care International; 2013.

40. Con la medicina multicultural se amplía la cobertura sanitaria: Narro. La Jornada [Internet]. 9 abr 2016 [citado 26 may 2016]. Disponible en: https://goo.gl/Jurm8w.

41. Barboza C, Carbajal B. En salud, educación y agro, 50\% del recorte. Milenio [Internet]. 25 jun 2016 [citado 28 ago 2016]. Disponible: https:// goo.gl/P4Fwi5.

42. Zavala destaca ante la OMS enfoque intercultural en salud. El Economista [Internet]. 28 feb 2012 [citado 26 may 2016]. Disponible en: https:// goo.gl/KXEuMH.
43. Brachet-Márquez V. Salud y seguridad social, 1917-2008: ¿Quién decide? In: Méndez JL. (ed.). Los grandes problemas de México. México: Colegio de México; 2010.

44. Colmenares-Roa $T$, Cervantes $L$, Ruesga $M$, Lino-Pérez L, Campos-Navarro R, Peláez-Ballestas I. Sociodemographic and clinical overview of the indigenous population admitted to the Hospital General de México "Dr. Eduardo Liceaga". Revista Médica del Hospital General de México. 2016;80(1):3-15.

45. Mandujano I. Simojovel: Los mató la miseria. Proceso [Internet]. 16 may 2015 [citado 26 may 2016]. Disponible en: https://goo.gl/YHBqto.

46. Pérez-Alonso JA. Oaxaca, paro en el hospital Valdivieso. La Jornada [Internet] 13 may 2015 [citado 26 may 2016]. Disponible en: https://goo. $\mathrm{gl} /$ FhMezS.

47. Cuyul A. Salud intercultural y la patrimonialización de la salud mapuche en Chile. In: Comunidad de Historia Mapuche. Ta iñ fijke xipa raquizuameluwün: Historia, colonialismo y resistencia desde el país Mapuche. Temuco: Ediciones de la Comunidad de Historia Mapuche; 2012.

48. Ramírez-Hita S. La interculturalidad sin todos sus agentes sociales: El problema de la salud intercultural en Bolivia. In: Fernández G. (comp.). Salud e interculturalidad en América latina: Antropología de la salud y crítica intercultural. Quito: Ediciones AbyaYala, AECl; 2006.

49. Ramírez-Hita S. Salud intercultural: Crítica y problematización a partir del contexto boliviano. La Paz: Instituto Superior Ecuménico Andino de Teología; 2011.

50. Peña EY, Hernández-Albarrán L. Recursos curativos y patrimonio biocultural en Suchitlán, Comala, Colima. México: Instituto Nacional de Antropología e Historia; 2014. 